\title{
Primary Hepatic Angiosarcoma Revealed by a Hemorrhagic Parietal Metastasis about a Case in Lomé
}

\author{
Mamadou Diallo', Mazamaesso Tchaou ${ }^{1 *}$, Issifou Boube1, Lantam Sonhaye ${ }^{2}$, Komlavi Adjenou ${ }^{2}$ \\ ${ }^{1}$ Department of Radiology, Sylvanus Olympio University Teaching Hospital of Lomé, Lomé, Togo \\ ${ }^{2}$ Department of Radiology, Campus University Teaching Hospital, Lomé, Togo \\ Email: mamadiallo126@yahoo.fr, *joseph_tchaou@yahoo.fr, iboube35@gmail.com, sonhayelantam@gmail.com, \\ kadjenou@yahoo.fr
}

How to cite this paper: Diallo, M., Tchaou, M., Boube, I., Sonhaye, L. and Adjenou, K. (2019) Primary Hepatic Angiosarcoma Revealed by a Hemorrhagic Parietal Metastasis about a Case in Lomé. Open Journal of Radiology, 9, 176-180. https://doi.org/10.4236/ojrad.2019.93016

Received: August 10, 2019

Accepted: September 13, 2019

Published: September 16, 2019

Copyright () 2019 by author(s) and Scientific Research Publishing Inc. This work is licensed under the Creative Commons Attribution International License (CC BY 4.0).

http://creativecommons.org/licenses/by/4.0/

\begin{abstract}
Angiosarcoma is a malignant tumor developed at the expense of endothelial cells. Primary hepatic localization is rare. Chronic toxic exposure is classically described as responsible for the development of this tumor. The diagnosis is based on the pathological examination. The authors report a case of hepatic angiosarcoma in a 28-year-old woman who received for a thoraco-abdominal hemorrhagic parietal secondary lesion. A thoracic abdominal and parietal ultrasound was performed followed by thoraco-abdomino-pelvic CT. The diagnosis was confirmed by histology. Palliative treatment combining surgery and chemotherapy has been established with a poor prognosis.
\end{abstract}

\section{Keywords}

Hepatic Angiosarcoma, Ultrasound, CT-Scan, Metastasis, Histology

\section{Introduction}

Angiosarcoma is a vascular tumor developed from endothelial cells. It can be seen in different locations. The most common sites are skin and soft tissues [1]. The primary hepatic localization of angiosarcoma is rare, and represents only $4 \%$ of angiosarcomas from all locations combined, and $1.8 \%$ of primary hepatic malignancies [2] [3].

We report a case of metastatic primary hepatic angiosarcoma with analysis of diagnostic findings and therapeutic means.

\section{Case Report}

Patient X, 28 years old, with no specific antecedent, presents right thoraco- 
abdominal wall swelling (Figure 1), bleeding with moderate abdominal pain. This symptomatology had been evolving for two months and gradually worsening. There was no notion of occupational intoxication. Hepatic serology B was negative.

A thoraco-abdominal and parietal ultrasound was performed followed by thoraco-abdomino-pelvic CT. On ultrasound, there was a large heterogeneous hypoechoic hepatic mass, with irregular contours, hyper vascularized with color Doppler (Figure 2). This formation was associated with another right parietal mass adjacent to the liver which had the same characteristic as the hepatic lesion. CT showed heterogeneous hepatic lesion of the segments VI and VII, with irregular limits and poor enhancement after contrast injection, connected to the parietal mass by a pedicle (Figure 3). The two hypothetic radiological diagnosis prior to biopsy were hepatic hemangioma and angiosarcoma.

In order to stop the haemorrhage, the parietal mass was resected surgically with histological exam. This exam evoked a grade II angiosarcoma according to FNCLCC. A week later, another preoperative abdomino-pelvic CT was performed, which showed the appearance of multiple secondary intrahepatic lesions on the left liver lobe associated with intra-peritoneal fluid collection, pleural, pulmonary and bone metastasis (Figure 4). Chemotherapy was introduced. The patient dead six months after the diagnosis.

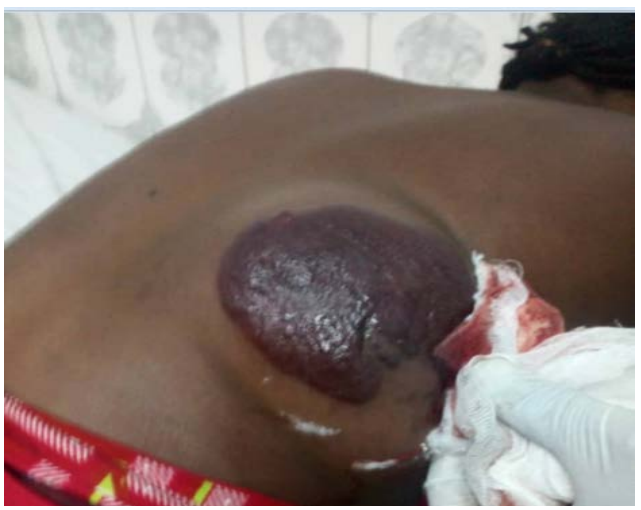

Figure 1. Photograph of the parietal lesion.

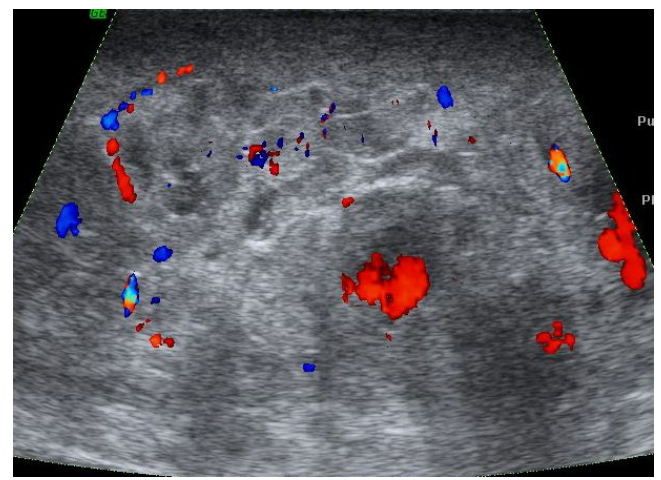

Figure 2. Ultrasound image of the hepatic mass, heterogeneous hyperechoic and vascularized with color Doppler. 


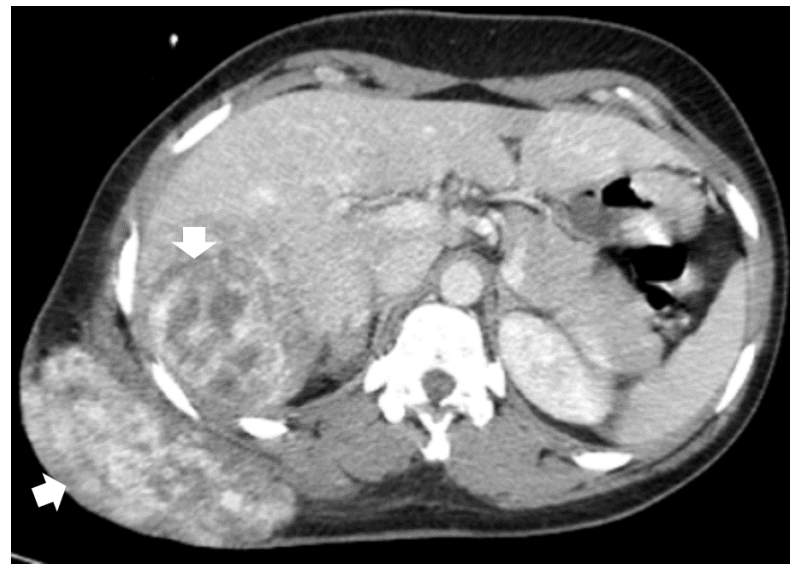

Figure 3. Abdominal CT showing hepatic lesion connected to the parietal mass (arrows) by a pedicle.

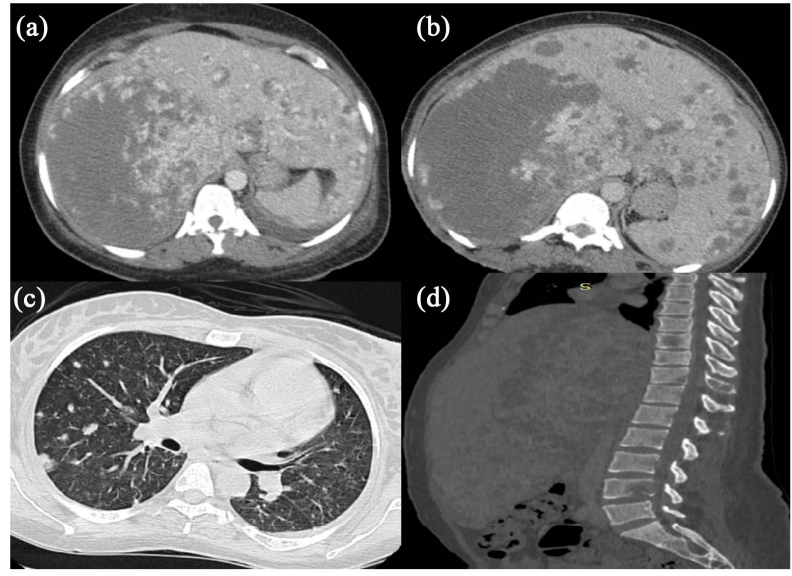

Figure 4. Thoraco-abdomial CT performed one month after removal of the parietal lesion showing lesion progression with hepatic (a) and (b), pulmonary (c) and bone (d) metastases.

\section{Discussion}

Angiosarcoma is a rare vascular tumor $(<0.1 / 100,000)$, and represents less than $2 \%$ of primary liver tumors. For this case, the clinical, radiological, histological and therapeutic data were analyzed. The patient was 28 years old. These demographics are different from data in the literature that describe a male predominance with a sex ratio of 3:1 [2] and a mean age of 60 years. The clinical symptoms revealing this tumor are varied and not specific, such as abdominal pain, fever, or deterioration of the general condition [2] [4]. The search for exposure to carcinogens of the arsenic and vinyl chloride type should be systematic. For our observation, no exposure to carcinogens was found. Hepatic dysfunction may also induce bleeding disorders, particularly as in the case of our patient, who was admitted for thoraco-abdominal wall hemorrhage with respect to the hepatic lesion.

Radiologically, the diagnosis of primary hepatic angiosarcoma remains difficult and raises the problem of differential diagnoses, such as other vascular tu- 
mors and other malignant tumors. On the CT scan, hepatic lesions of segments VI and VII were heterogeneous, with irregular limits with poor and heterogeneous enhancement kinetics. These CT data are globally identical to the literature which notes one or more heterogeneous liver lesions, with hyperdensity possibly corresponding to internal haemorrhagic reorganization and heterogeneous and more rarely centripetal enhancement after intravenous injection of iodinated contrast medium [2] [3] [5] [6]. In our case, the tumor is unique in comparison with the recent Chinese series which reports only solitary tumors [2]. In order of frequency, the secondary locations concern the lung and then the spleen [5], unlike our observation where the secondary locations are parietal, hepatic, lung and bone.

In our case, for histopathology, samples were taken from the parietal lesion contrary to the data of the literature where the sample concerned the hepatic lesion. This difference is explained not only by the accessibility of the parietal lesion but also by the hemorrhagic risk which may worsen its general condition in case of liver biopsy. The histological study confirmed the diagnosis of hepatic angiosarcoma in accordance with the data of the literature [7].

Therapeutically, a surgical excision of the parietal mass was performed for the purpose not only hemostatic but also for histological study. Similarly, the chemotherapy initiated since histological confirmation did not lead to a significant improvement in the clinical status of the patient, in correlation with the data found in the literature [1] [8] [9]. The median survival is 5 months [10], however, in our case history taking, the patient survived six months.

\section{Conclusion}

Primary hepatic angiosarcoma is a rare tumor. The diagnosis is based on clinical diagnosis and radiology and the diagnosis of certainty is histological. Surgical excision, recognized as the treatment of choice of first intention is rarely feasible because of the late diagnosis and multifocal lesions. Therapeutic alternatives based on chemotherapy have shown only partial efficacy and the prognosis remains poor.

\section{Consent for Publication}

Consent of the father of the patient has been obtained for publication of this case.

\section{Authors' Contribution}

MD and MT was responsible for the design of the study, undertook the field study, performed data collection, analysis and interpretation, and wrote the manuscript.

IB, LS and KA: Participated in the design of the report of the case and preparation of the final manuscript.

All authors have read and approved the final manuscript to be submitted for publication. 


\section{Conflicts of Interest}

The authors report no conflicts of interest.

\section{References}

[1] Fayette, J., Martin, E., Piperno-Neumann, S., Le Cesne, A., Robert, C., Bonvalot, S., et al. (2007) Angiosarcomas, a Heterogeneous Group of Sarcomas with Specific Behavior Depending on Primary Site: A Retrospective Study of 161 Cases. Annals of Oncology, 18, 2030-2036. https://doi.org/10.1093/annonc/mdm381

[2] Duan, X.F. and Li, Q. (2012) Primary Hepatic Angiosarcoma: A Retrospective Analysis of 6 Cases. Journal of Digestive Diseases, 13, 381-385. https://doi.org/10.1111/j.1751-2980.2012.00600.x

[3] Park, Y.S., Kim, J.H., Kim, K.W., Lee, I.S., Yoon, H.K., Ko, G.Y., et al. (2009) Primary Hepatic Angiosarcoma: Imaging Findings and Palliative Treatment with Transcatheter Arterial Chemoembolization or Embolization. Clinical Radiology, 64, 779-785. https://doi.org/10.1016/j.crad.2009.02.019

[4] Huang, N.C., Wann, S.R., Chang, H.T., Lin, S.L., Wang, J.S. and Guo, H.R. (2011) Arsenic, Vinyl Chloride, Viral Hepatitis, and Hepatic Angiosarcoma: A Hospital-Based Study and Review of Literature in Taiwan. BMC Gastroenterology, 11, Article No. 142. https://doi.org/10.1186/1471-230X-11-142

[5] Koyama, T., Fletcher, J.G., Johnson, C.D., Kuo, M.S., Notohara, K. and Burgart, L.J. (2002) Primary Hepatic Angiosarcoma: Findings at CT and MR Imaging. Radiology, 222, 667-673. https://doi.org/10.1148/radiol.2223010877

[6] Delabrousse, E., Valmary-Degano, S., Aubry, S., Dietsch, E. and Badet, N. (2013) TDM des tumeurs abdominales. Elsevier, Paris.

[7] Cazorla, A., et al. (2014) L'angiosarcome hépatique primitif: Etude rétrospective de huit cas. Annalesde Pathologie, 34, 462-468.

https://doi.org/10.1016/j.annpat.2014.10.004

[8] Nazir, Z. and Pervez, S. (2006) Malignant Vascular Tumors of Live in Neonates. Journal of Pediatric Surgery, 41, e49-e51. https://doi.org/10.1016/j.jpedsurg.2005.10.078

[9] Agulnik, M., Yarber, J.L., Okuno, S.H., von Mehren, M., Jovanovic, B.D., Brockstein, B.E., et al. (2013) An Open-Label, Multicenter, Phase II Study of Bevacizumab for the Treatment of Angiosarcoma and Epithelioid Hemangioendotheliomas. Annals of Oncology, 24, 257-263. https://doi.org/10.1093/annonc/mds237

[10] Sanya, W., et al. (2017) Hepatic Angiosarcoma with Clinical and Histological Features of Kasabach-Merritt Syndrome. World Journal of Gastroenterology, 23, 2443-2447. https://doi.org/10.3748/wjg.v23.i13.2443 